\title{
Adverse Drug Reactions from Real-World Data in Inflammatory Bowel Disease Patients in the IBDREAM Registry
}

\author{
Eline L. Giraud ${ }^{1}$ · Pepijn W. A. Thomas ${ }^{2}{ }^{\oplus}$. Jette A. van Lint ${ }^{1}$ - Eugene P. van Puijenbroek ${ }^{1}$. Tessa E. H. Römkens ${ }^{3}$. \\ Rachel L. West ${ }^{4} \cdot$ Maurice G. V. M. Russel $^{5}$. Jeroen M. Jansen ${ }^{6} \cdot$ Naomi T. Jessurun $^{1} \cdot$ Frank Hoentjen $^{2} \cdot$ IBDREAM \\ registry
}

Accepted: 15 January 2021 / Published online: 4 February 2021

(c) The Author(s) 2021

\begin{abstract}
Introduction Inflammatory bowel disease (IBD) frequently requires chronic immunosuppressive treatment and active involvement from patients during treatment decision making. Information about the risk of developing adverse drug reactions (ADRs) to IBD therapies is required in this process.

Objective The aim of this study was to describe the ADRs reported in IBD patients from real-world data, using the Dutch nationwide IBDREAM registry, and compare the occurrence and cumulative incidences with the Summary of Product Characteristics (SmPC) of the associated drugs.

Methods In this retrospective multicentre study, ADRs related to IBD medication were assessed. Only reports associated with the use of drugs used for the maintenance treatment of IBD were included. All ADRs were verified by healthcare professionals and coded by trained pharmacovigilance assessors.

Results In total, 3080 ADRs were reported in 1179 patients. Twenty-three new drug-ADR associations related to the use of azathioprine, mercaptopurine, infliximab, oral mesalamine and thioguanine were reported in the IBDREAM registry that were not mentioned in the corresponding SmPCs. The most frequently reported new association was pyrexia for azathioprine (3.1\%) and mercaptopurine (4.9\%). In addition, there were seven ADRs with a higher cumulative incidence in IBDREAM compared with the SmPC, and included, among others, arthralgia during mercaptopurine use (2.5\%), and diarrhoea (1.4\%), alopecia (1.2\%) and infections (1.6\%) during azathioprine use.

Conclusions Based on real-world data, ADR reporting demonstrated new ADRs and higher incidences of ADRs to IBD therapies. This information will contribute to drug safety by updating the SmPCs, allowing better risk assessment and communication towards patients.
\end{abstract}

Eline L. Giraud, Pepijn W. A. Thomas have contributed equally to this manuscript.

\section{Pepijn W. A. Thomas}

pepijn.thomas@radboudumc.nl

1 Netherlands Pharmacovigilance Centre Lareb, 's-Hertogenbosch, The Netherlands

2 Department of Gastroenterology and Hepatology, Radboud University Medical Centre, Nijmegen, The Netherlands

3 Department of Gastroenterology and Hepatology, Jeroen Bosch Ziekenhuis, 's-Hertogenbosch, The Netherlands

4 Department of Gastroenterology and Hepatology, Franciscus Gasthuis en Vlietland, Rotterdam, The Netherlands

5 Department of Gastroenterology and Hepatology, Medisch Spectrum Twente, Enschede, The Netherlands

6 Department of Gastroenterology and Hepatology, Onze Lieve Vrouwe Gasthuis, Amsterdam, The Netherlands

\section{Introduction}

Inflammatory bowel disease (IBD), encompassing ulcerative colitis (UC), Crohn's disease (CD) and IBD unclassified (IBD-U), is characterised by a chronic relapsing and remitting inflammation of the digestive tract [1,2]. Immunosuppressants and biologicals are used to induce and maintain remission in IBD [3]. The selection of therapy is based on several factors, including location and severity of inflammation, previous treatment response, possible adverse drug reactions (ADRs) and patient preferences. As IBD requires lifelong treatment, patients are actively involved in treatment decision making and are informed about the risks and benefits of all treatment options, including possible ADRs.

Current drug safety profiles may not reflect the complete spectrum of ADRs as provided in the Summary of Product 


\section{Key Points}

Through the use of real-world adverse drug reaction (ADR) data from an inflammatory bowel disease (IBD) patient registry, we found 24 new drug-ADR associations related to the use of IBD maintenance medication.

The most frequently reported new drug-ADR association was pyrexia for azathioprine $(3.1 \%)$ and mercaptopurine $(4.9 \%)$.

We found several higher cumulative ADR incidence rates in the patient registry compared with the corresponding Summary of Product Characteristics (SmPCs).

Real-world ADR data from patient registries provide an added value on incidences of ADRs compared with information in the SmPCs.

The findings of this study will contribute to drug safety by updating the SmPCs, allowing better risk assessment and communication towards patients.

Characteristics (SmPC). The SmPC is the summary of data derived from clinical trials, post-authorisation safety studies and spontaneous reports [4], and provides information for patient information leaflets (PILs). Both the SmPC and the PIL are used to inform patients and healthcare professionals (HCPs) on drug safety. Therefore, information on ADRs in the SmPC should match with ADRs observed in daily practice. However, as the information in the SmPC is based on controlled circumstances of (pre)clinical trials, both the SmPC and PIL may not give an accurate overview of the type and incidence of ADRs in the daily clinical setting. In turn, it may be challenging to choose the most favourable drug for each patient considering individual factors such as comorbidities and comedication.

Nationwide registries collecting real-world data about ADRs may be used to enlarge the SmPC and for future updates on postmarketing surveillance of drugs. The IBDREAM registry is a multicentre registry specifically developed for IBD patients treated in The Netherlands [5]. ADRs that are registered in IBDREAM are directly, electronically forwarded to the Netherlands Pharmacovigilance Centre (Lareb) [6]. Information about real-world ADR incidences may provide a better understanding of possible risks of treatment options for both patients and HCPs, which may improve clinical care of IBD patients. Subsequently, this may improve clinical care of IBD patients in addition to other factors such as personal risk factors and the course of an ADR. Therefore, the aim of this study was to describe the frequency of HCP-reported ADRs in IBD patients from realworld data, using the IBDREAM registry, and compare this with the SmPCs of the associated therapies. Furthermore, secondary aims were to describe new drug-ADR associations that were not included in the current SmPCs, and incidences of ADRs different from or unknown in the SmPCs.

\section{Methods}

\subsection{Study Design and Patient Population}

In this retrospective multicentre cohort study, we described the frequency of ADRs reported in IBD patients from realworld data collected in the IBDREAM registry, and compared this with data from clinical trials, post-authorisation marketing studies, and spontaneous reports, summarised in the SmPCs (see Fig. 1). All included patients had a clinical diagnosis of $\mathrm{CD}$, UC or IBD-U according to international guidelines. We assessed all ADR reports related to IBD medication originating from the IBDREAM registry that were reported to Lareb. ADRs were included if they were associated with the use of drugs indicated for maintenance treatment of IBD: adalimumab, azathioprine, golimumab, infliximab, mercaptopurine, mesalamine, methotrexate, thioguanine, ustekinumab and vedolizumab. For methotrexate, ADRs were analysed separately for oral and subcutaneous administration. For mesalamine, ADRs were analysed separately for either oral administration or rectal administration. The cumulative incidences of the ADRs were calculated and compared with the associated SmPCs. Therefore, a total of 12 SmPCs were used in this study [9-20]. All ADRs reported up to 24 July 2020 were collected.

\subsection{Data Source and Processing}

Data were retrieved from the IBDREAM registry, a multicentre IBD registry in The Netherlands that prospectively collects medical data, including ADRs, from IBD patients in daily practice in five hospitals in The Netherlands [5]. Enrolled patients received a personal and secure account within the IBDREAM registry, allowing them to report ADRs to their HCP. Within IBDREAM, patients can select one of the used drugs and report ADRs related to this specific drug. These ADRs were verified by the HCP and recorded in the IBDREAM registry. In addition, ADRs were retrieved from the electronic healthcare records or directly recorded by the HCP during an outpatient visit. All ADRs were entered in an open text field. Thus, both patients and HCPs may report ADRs, while all ADRs were verified and registered by HCPs.

For this study, all included ADR reports were directly forwarded to Lareb for analysis. Subsequently, the ADRs were coded by trained pharmacovigilance assessors of Lareb according to the Medical Dictionary for Regulatory 


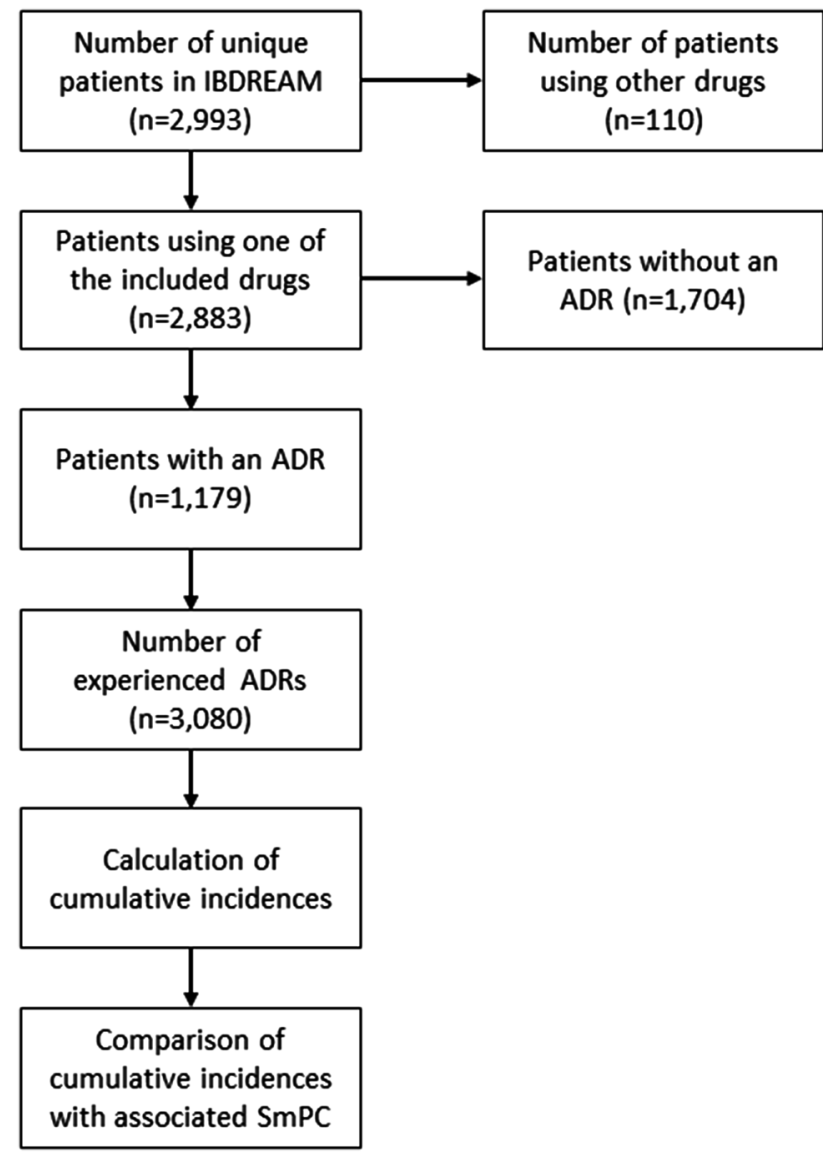

Fig. 1 Study design. ADRs adverse drug reactions, IBDREAM Inflammatory Bowel Disease Registry, SmPC Summary of Product Characteristics

Activities (MedDRA) codes, version 20.1 [7]. MedDRA contains five hierarchical grouping levels, ranging from a very general description to a very specific description. The most specific level is the 'Lowest Level Term' (LLT), which is linked to only one 'Preferred Term' (PT). PTs are then grouped into 'High Level Terms' (HLTs) based on anatomy, aetiology and function. HLTs are consecutively linked to 'High Level Group Terms' (HLGTs) and finally grouped into 'System Organ Classes' (SOCs) based on either aetiology, manifestation site or purpose. In this study, PTs were used to describe ADRs. The following data from IBDREAM were retrieved and used: date of birth, sex, age, drugs, product name, route of administration, dosage, start and stop dates of the drug, description of the ADR, start date of the ADR, change in treatment due to the ADR, ADR as LLT, PT, HLT, HLGT and SOC, and hospital source.

In the current study, the SmPCs of the included drugs were used as a reference. These SmPCs were extracted from the online database of the Dutch Medicines Evaluation Board [8]. As every drug product has its own SmPC, the most recently revised version of each drug was used as a reference in this study. In each SmPC, known ADRs are tabulated per SOC per frequency category and can be found in Sect. 4.8. The frequency categories are described as 'very common' ( $\geq 10 \%)$, 'common' ( $\geq 1 \%$ to $<10 \%$ ), 'uncommon' $(\geq 0.1 \%$ to $<1 \%)$, 'rare' $(\geq 0.01 \%$ to $<0.1 \%)$, 'very rare' $(<0.01 \%)$ or unknown.

\subsection{Data Analysis}

Baseline characteristics are presented as mean with standard deviation (SD) or frequency with percentage (\%). For all ADRs included in this study, cumulative incidences were calculated comparable to the frequency measure used in the SmPCs. If a specific drug-ADR association was reported more than once in the same patient, all individual ADR reports were included in the analysis. However, an intended rechallenge, which was performed by HCPs to verify a drug-ADR association, was not considered a new case report. HCPs did not report any intended rechallenges in IBDREAM. Subsequently, all calculated cumulative incidences were described and compared with the corresponding SmPC. Time-to-onset of the ADR was calculated in days between the start date of the drug and start date of the ADR. Data analysis was performed using SPSS 25.0 (IBM Corporation, Armonk, NY, USA).

\subsection{Data Presentation}

We described the cumulative incidences of reported ADRs in the IBDREAM registry that were compared with the incidences in the corresponding SmPCs. All new drug-ADR associations with 10 or more case reports registered in the IBDREAM registry, but not mentioned in the SmPC, were tabulated. These were only included if they had not been described in any other SmPC of the related drug. Subsequently, all ADRs with a higher cumulative incidence in the IBDREAM registry compared with the corresponding SmPC, and with 10 or more case reports, were described. Finally, all ADRs with an unknown cumulative incidence in the SmPC but a known incidence in the IBDREAM registry, and with 10 or more case reports, were described. In addition, all ADRs stratified by drug are described in detail in the electronic supplementary material, including cumulative incidences.

\subsection{Ethical Considerations}

The study protocol was approved by the Radboudumc Medical Ethical Committee [2015-2245], and all patients signed written informed consent. 


\section{Results}

\subsection{Baseline Characteristics}

From the 2993 patients included in the IBDREAM registry, a total of 2883 (96.3\%) used one of the included drugs in the current study. A total of 3080 ADRs were reported in 1179 patients. Baseline characteristics are presented in Table 1. Overall, $40.9 \%$ of all patients who used one of the included drugs experienced one or more ADRs. Most ADRs were experienced by mercaptopurine users (41.9\%) and the least ADRs were experienced by rectal mesalamine users $(4.8 \%)$. Fewer UC patients (35.6\%) than CD patients $(62.3 \%)$ included in the IBDREAM registry used one of the included IBD drugs. The mean age was 45.1 years $( \pm 15.7)$ and slightly more patients were female (56.0\%) than male. Patients who experienced ADRs were more often female (60.4\%). Of all included ADRs, the drug was withdrawn in $73.4 \%$ of cases and the dose was reduced in $1.9 \%$ of cases. In $3.1 \%$ of ADRs, the dose was not changed and in $22.1 \%$ no information was provided about a change in treatment. More specifically, the withdrawal rate due to an ADR per drug ranged from $7.4 \%$ for golimumab to $55.3 \%$ for mercaptopurine. These withdrawal rates are described in Electronic Supplementary Table 2 .

\subsection{Comparison Cumulative Incidences in IBDREAM versus the Summary of Product Characteristics (SmPCs)}

A total of 3080 ADRs were analysed, encompassing a total of 390 unique ADRs. The SmPC of thioguanine reports only a few ADRs, resulting in the least comparisons made between the calculated cumulative incidences of the ADRs attributed to the use of thioguanine compared with the corresponding SmPC. All ADRs, including their cumulative incidences, stratified by drug, are tabulated in Electronic Supplementary Table 1 .

\subsection{New Drug-Adverse Drug Reaction (ADR) Associations}

A total of 23 new drug-ADR associations with 10 or more case reports were reported in the IBDREAM registry that were not mentioned in the corresponding SmPCs (Table 2).

Table 1 Baseline characteristics

\begin{tabular}{llll}
\hline & $\begin{array}{l}\text { IBDREAM Registry patients who } \\
\text { used one of the included drugs }\end{array}$ & $\begin{array}{l}\text { Unique patients } \\
\text { with ADRs }{ }^{\mathrm{a}}\end{array}$ & Reported ADRs \\
\hline Total & $2883(100)$ & $1179(100)$ & $3080(100)$ \\
Age, years [mean \pm SD] & $45.1 \pm 15.7$ & $45.9 \pm 15.4$ & $39.5 \pm 15.2$ \\
Sex, female & $1614(56.0)$ & $714(60.4)$ & $1883(61.1)$ \\
IBD type & & & \\
Crohn's disease & $1796(62.3)$ & $821(69.6)$ & $2139(69.4)$ \\
Ulcerative colitis & $1026(35.6)$ & $329(27.9)$ & $822(26.7)$ \\
IBD-U & $61(2.1)$ & $29(2.5)$ & $119(3.9)$ \\
Drug used & & & \\
Mesalamine oral & $1423(49.4)$ & $156(13.2)$ & $274(8.9)$ \\
Infliximab & $1407(48.8)$ & $299(25.4)$ & $486(15.8)$ \\
Azathioprine & $1399(48.5)$ & $518(43.9)$ & $778(25.3)$ \\
Mercaptopurine & $1104(38.3)$ & $463(39.3)$ & $788(25.6)$ \\
Adalimumab & $945(32.8)$ & $150(12.7)$ & $209(6.8)$ \\
Tioguanine & $568(19.7)$ & $144(12.2)$ & $204(6.6)$ \\
Mesalamine rectal & $525(18.2)$ & $25(2.1)$ & $47(1.5)$ \\
Vedolizumab & $319(11.1)$ & $30(2.5)$ & $49(1.6)$ \\
Methotrexate SC & $204(7.1)$ & $56(4.7)$ & $103(3.3)$ \\
Ustekinumab & $192(6.7)$ & $48(4.1)$ & $92(3.0)$ \\
Methotrexate oral & $138(4.8)$ & $28(2.4)$ & $38(1.2)$ \\
Golimumab & $27(0.9)$ & $5(0.4)$ & $12(0.4)$ \\
\hline
\end{tabular}

Data are expressed as $n(\%)$ unless otherwise specified

$A D R s$ adverse drug reactions, IBDREAM Inflammatory Bowel Disease Registry, IBD inflammatory bowel disease, $I B D-U$ inflammatory bowel disease unclassified, $S D$ standard deviation, $S C$ subcutaneous

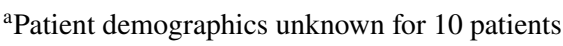

${ }^{\mathrm{b}}$ Patient demographics unknown for 10 patients (14 ADRs) 
Most new drug-ADR associations with a high number of case reports were associated with the use of azathioprine and mercaptopurine. The new drug-ADR associations with azathioprine and mercaptopurine were similar, including pyrexia, abdominal pain, malaise, fatigue and headache.

\subsection{ADRs with a Higher Incidence Compared with the SmPC}

A total of seven ADRs with 10 or more case reports were reported, with a higher cumulative incidence in IBDREAM compared with the corresponding SmPCs (Table 3); these were most often associated with the use of azathioprine and mercaptopurine. Arthralgia associated with the use of mercaptopurine, autoantibody positive associated with the use of infliximab and diarrhoea, and alopecia and viral, fungal and bacterial infections associated with the use of azathioprine were all reported with a 'common' $(\geq 1 \%$ to $<10 \%)$ cumulative incidence in the IBDREAM registry, whereas these were all mentioned in the SmPC with an uncommon $(\geq 0.1 \%$ to $<1 \%)$ incidence. Skin rash and alopecia associated with the use of mercaptopurine were also reported with a 'common' $(\geq 1 \%$ to $<10 \%)$ cumulative incidence in IBDREAM but were mentioned with a 'rare' $(\geq 0.01 \%$ to $<0.1 \%)$ cumulative incidence in the SmPC.

\subsection{ADRs with an Unknown Incidence in the SmPC}

Malaise associated with the use of thioguanine was reported in IBDREAM, with a cumulative incidence of $2.6 \%$

$(n=15)$, which was classified as common $(\geq 1 \%$ to $<10 \%)$. This is the only ADR with 10 or more case reports that was mentioned with an unknown incidence in the corresponding SmPC.

\section{Discussion}

In this retrospective multicentre cohort study, we described a total of 23 new drug-ADR associations that were not mentioned in the SmPC. In addition, a total of seven ADRs were reported with a higher cumulative incidence in the IBDREAM registry compared with the corresponding SmPC. Finally, there was one ADR that was mentioned with
Table 2 New drug-ADR associations (with 10 or more case reports) from the IBDREAM registry that were not mentioned in the SmPC

\begin{tabular}{|c|c|c|c|c|}
\hline Drug & $\mathrm{ADR}$ as $\mathrm{PT}$ & $\begin{array}{l}\text { Incidence in } \\
\text { IBDREAM } \\
(\%)\end{array}$ & $\begin{array}{l}\text { No. of } \\
\text { case } \\
\text { reports }\end{array}$ & $\begin{array}{l}\text { Time-to-onset of ADR, } \\
\text { days [median (IQR)] }\end{array}$ \\
\hline \multirow[t]{8}{*}{ Azathioprine $(n=1399)$} & Pyrexia & 3.1 & 45 & $22(11-42)$ \\
\hline & Abdominal pain & 2.9 & 41 & $28(1-47)$ \\
\hline & Malaise & 2.1 & 30 & $26(8-369)$ \\
\hline & Pruritus & 2.1 & 29 & $112(45-386)$ \\
\hline & Arthralgia & 1.9 & 27 & $122(26-366)$ \\
\hline & Fatigue & 1.0 & 14 & $53(298-228)$ \\
\hline & Headache & 0.9 & 12 & $26(15-41)$ \\
\hline & Amylase increased & 0.7 & 10 & $25(16-35)$ \\
\hline Infliximab $(n=1407)$ & Malaise & 0.9 & 13 & $123(7-225)$ \\
\hline \multirow[t]{9}{*}{ Mercaptopurine $(n=1104)$} & Pyrexia & 4.9 & 54 & $19(13-25)$ \\
\hline & Abdominal pain & 4.4 & 49 & $30(19-58)$ \\
\hline & Malaise & 4.2 & 46 & $20(13-30)$ \\
\hline & Fatigue & 2.6 & 29 & $36(19-60)$ \\
\hline & Headache & 2.5 & 28 & $21(11-46)$ \\
\hline & Diarrhoea & 1.5 & 17 & $23(18-29)$ \\
\hline & Myalgia & 1.5 & 16 & $18(10-73)$ \\
\hline & Decreased appetite & 1.3 & 14 & $49(14-80)$ \\
\hline & Drug level increased & 0.9 & 10 & $69(61-235)$ \\
\hline Mesalamine tablets $(n=1423)$ & Malaise & 0.8 & 11 & $15(6-72)$ \\
\hline \multirow[t]{4}{*}{ Thioguanine $(n=568)$} & Nausea & 4.8 & 27 & $31(22-113)$ \\
\hline & Abdominal pain & 3.2 & 18 & $21(5-65)$ \\
\hline & Arthralgia & 1.9 & 11 & $43(14-295)$ \\
\hline & Rash & 1.8 & 10 & $163(77-193)$ \\
\hline
\end{tabular}

$A D R$ adverse drug reaction, IBDREAM Inflammatory Bowel Disease Registry, SmPC Summary of Product Characteristics, $P T$ preferred term, IQR interquartile range 
Table 3 ADRs with a higher cumulative incidence in the IBDREAM registry compared with the corresponding SmPCs

\begin{tabular}{|c|c|c|c|c|c|}
\hline Drug & $\mathrm{ADR}$ as $\mathrm{PT}$ & Incidence in the SmPC & $\begin{array}{l}\text { Incidence in } \\
\text { IBDREAM }(\%)\end{array}$ & $\begin{array}{l}\text { No. of case } \\
\text { reports }\end{array}$ & $\begin{array}{l}\text { Time-to-onset of ADR, } \\
\text { days [median (IQR)] }\end{array}$ \\
\hline \multirow[t]{3}{*}{ Azathioprine } & $\begin{array}{l}\text { Viral, fungal and bacterial } \\
\text { infections }\end{array}$ & $\begin{array}{l}\text { 'Uncommon' } \\
\geq 0.1 \% \text { to }<1 \%\end{array}$ & 1.6 & 23 & $332(30-1089)$ \\
\hline & Diarrhoea & $\begin{array}{l}\text { 'Uncommon' } \\
\geq 0.1 \% \text { to }<1 \%\end{array}$ & 1.4 & 20 & $19(9-44)$ \\
\hline & Alopecia & $\begin{array}{l}\text { 'Uncommon' } \\
\geq 0.1 \% \text { to }<1 \%\end{array}$ & 1.2 & 17 & $168(20-392)$ \\
\hline Infliximab & Autoantibody positive & $\begin{array}{l}\text { 'Uncommon' } \\
\geq 0.1 \% \text { to }<1 \%\end{array}$ & 1.4 & 20 & $147(77-462)$ \\
\hline \multirow[t]{3}{*}{ Mercaptopurine } & Arthralgia & $\begin{array}{l}\text { 'Uncommon' } \\
\geq 0.1 \% \text { to }<1 \%\end{array}$ & 2.5 & 28 & $21(16-50)$ \\
\hline & Skin rash & $\begin{array}{l}\text { 'Rare' } \\
\geq 0.01 \% \text { to }<0.1 \%\end{array}$ & 1.7 & 19 & $26(18-49)$ \\
\hline & Alopecia & $\begin{array}{l}\text { 'Rare' } \\
\geq 0.01 \% \text { to }<0.1 \%\end{array}$ & 1.1 & 12 & $52(27-158)$ \\
\hline
\end{tabular}

$A D R$ adverse drug reactions, IBDREAM Inflammatory Bowel Disease Registry, SmPC Summary of Product Characteristics, $P T$ preferred term, $I Q R$ interquartile range

an unknown incidence in the SmPC, for which we calculated a cumulative incidence in IBDREAM.

Most new drug-ADR associations that were found in this study were related to azathioprine, such as pyrexia, malaise, arthralgia, nausea and diarrhoea. Although these symptoms are mentioned in the SmPC as part of a hypersensitivity reaction [10], our study suggests that they may also occur independently and may not always point towards a hypersensitivity reaction. Similarly, arthralgia, pyrexia and rash associated with the use of mercaptopurine are only reported in the SmPC as part of a hypersensitivity reaction [12], and not separately. These findings were supported by recent studies [21-24] in which pyrexia, malaise, nausea and diarrhoea associated with the use of azathioprine, and arthralgia, pyrexia and rash associated with the use of mercaptopurine, were reported separately. Symptoms such as arthralgia may also be considered underlying symptoms of IBD. Similarly, digestive intolerance such as diarrhoea, nausea, vomiting and abdominal pain may be due to other causes such as irritable bowel syndrome or underlying IBD and may therefore be potentially misclassified as an ADR despite careful assessment by HCPs and assessors during analysis. As we did not correct for the background incidence of these symptoms, the calculated cumulative incidences in our study might be an overestimation of true occurrence.

Skin rash associated with the use of mercaptopurine was observed with a 'common' $(\geq 1 \%$ to $<10 \%$ ) cumulative incidence in IBDREAM $(1.7 \%)$ while the ADR is documented as 'rare' $(\geq 0.01 \%$ to $<0.1 \%)$ in the SmPC. A retrospective study [25] reported a cumulative incidence of $3.0 \%$ for skin rash associated with the use of mercaptopurine in azathioprine-intolerant patients, suggesting a 'common' frequency, which is in line with the findings in our study.
There were four new ADRs associated with the use of thioguanine, including arthralgia. Arthralgia is a common idiosyncratic ADR during thioguanine use [26] and may occur at any time during the use of thioguanine. Arthralgia associated with the use of thioguanine was reported with a 'common' ( $\geq 1 \%$ to $<10 \%$ ) cumulative incidence in IBDREAM $(1.9 \% ; n=11)$. This finding is in line with the cumulative incidence of $5.8 \%$ reported in a Dutch retrospective study $(n=16)$ [27], while a Dutch prospective study reported an even higher cumulative incidence of 'very common', with $10.6 \%(n=10)$ [21]. From the latter 10 cases, seven were possibly related to arthralgia and three cases resulted in discontinuation. As stated previously, arthralgia is also associated with the use of azathioprine (cumulative incidence 1.9\%) and mercaptopurine (cumulative incidence $2.5 \%$ ). Patients who experience arthralgia during the use of azathioprine may benefit from a switch to mercaptopurine [28-30]. This might be due to nitro-imidazole, which is released from azathioprine when converted to mercaptopurine. In general, this would suggest a higher cumulative incidence of arthralgia during azathioprine use compared with mercaptopurine, which is in line with the findings in our study. However, other factors might also play a role in developing arthralgia, such as comorbidities and comedication. In addition, arthralgia is the most common extraintestinal manifestation of IBD, which occurs in onethird of IBD patients [31]. One notable finding in the current study is that the drug was withdrawn as a result of arthralgia in $66.7 \%(n=18)$ of the azathioprine cases, $75 \%(n=21)$ of the mercaptopurine cases and $100 \%(n=11)$ of the thioguanine cases. In all cases, patients reported resolution of symptoms, which points to a causal relationship. 
Nausea and abdominal pain associated with the use of thioguanine were reported with a 'common' cumulative incidence ( $4.8 \%$ and $3.2 \%$, respectively) in IBDREAM, but were not mentioned in the SmPC of thioguanine.

These findings were confirmed by a recent retrospective cohort study [27] that observed a 'common' cumulative incidence for both nausea and abdominal pain, with $2.2 \%$ $(n=6)$. In that study, patients experienced these ADRs as mild and the ADRs did not lead to treatment discontinuation.

The presence of antidrug antibodies directed at infliximab was reported with a higher cumulative incidence compared with the SmPC. However, antidrug antibodies themselves are usually not considered an ADR, and consequently the incidence rates of antidrug antibodies are underreported and underestimated in IBDREAM compared with the SmPC. Furthermore, ADRs that included investigations rather than symptoms (e.g. anaemia, leukocytosis) were only reported if this resulted in discontinuation of the treatment. In addition, only symptoms as a result of these type of ADRs were reported (e.g. fatigue in the context of anaemia). Therefore, the calculated cumulative incidences of these objective ADRs should be interpreted with caution as these are likely to be underestimations of cumulative incidences compared with the corresponding SmPCs.

This study shows that in the current system for ADR collection, as reflected by the SmPCs, there is room for improvement to provide a representative overview of the ADRs occurring in daily clinical practice. A previous study showed that HCPs significantly underreport ADRs [32]. HCPs may want to avoid the administrative burden of ADR reporting. In order to complement the information in the SmPC, other sources for ADR reporting, such as patient registries, should be considered. The advantages of these registries comprise the long follow-up per patient, the large number of patients included and the inclusion of patients who are normally not included in clinical trials due to specific selection criteria. Digital support for reporting and assessing ADRs is crucial to further facilitate this process.

A strength of this study is the use of real-world data from daily practice, including 2993 IBD patients. Because of the large number of patients treated in different hospitals across the country, the study population is a representative sample of IBD patients treated in The Netherlands. Therefore, the external validity of this study is high. Even though the ADRs were not assessed for causality, the HCP verified and registered all ADRs. Therefore, we believe that all drug-ADR combinations included in this study, especially the 23 new combinations, are strong drug-ADR associations that require further research.

A limitation of this retrospective observational study is that patients may not always report every complaint to their HCP. Additionally, HCPs will not always register every ADR reported by patients. These may both cause an underestimation of cumulative incidences reported in the IBDREAM registry compared with reality. Although we did not adjust for the background incidence and because some ADRs may be misclassified, the calculated cumulative incidences could be interpreted as the lower limit of the range of the related frequency category since the ADRs in this study were most often reported in case the drug was withdrawn $(75 \%)$ or the dosage was altered $(2 \%)$. These ADRs are therefore considered clinically relevant since they resulted in adjustment (dose reduction or discontinuation) of therapy. Second, the reported ADRs may also be a result of the underlying disease, co-medication or co-morbidities. Although careful assessment by the HCP and the pharmacovigilance assessor limits this risk, it cannot be excluded in all cases. The clinical impact (e.g. improvement of symptoms) of stopping therapy has facilitated this analysis to correctly assign ADRs to reported symptoms.

\section{Conclusions}

We report on the incidences of ADRs from a real-life registry, IBDREAM, which allowed us to update the ADR incidences, compared with SmPCs, to IBD medications. We observed 23 new drug-ADR associations with 10 or more case reports that are not mentioned in the corresponding SmPCs. Additionally, we reported ADRs that are more common than described in the SmPC, including infections, alopecia, arthralgia, skin rash and diarrhoea. These data will aid in well-informed clinical decision making on IBD therapies by patients and their physicians.

Supplementary Information The online version contains supplementary material available at https://doi.org/10.1007/s40264-021-01045-3.

\section{Declarations}

Funding Funding for the development and implementation of IBDREAM was obtained from the Netherlands Organisation for Health Research and Development (ZonMW, Grant number 836042002).

Conflicts of Interest Elaine L. Giraud, Pepijn W.A. Thomas, Jette A. van Lint, Eugene P. van Puijenbroek, Tessa E.H. Römkens, Maurice G.V.M. Russel, Jeroen M. Jansen, Naomi T. Jessurun, and Frank Hoentjen declare they have no conflicts of interest. Rachel L. West reports that she has participated in advisory boards and/or received financial compensation from Janssen and Pfizer, all outside the submitted work.

Ethics Approval The study protocol was approved by the Radboudumc Medical Ethical Committee [2015-2245].

Consent to Participate All patients signed written informed consent.

Consent for Publication All authors provided consent for publication. 
Data availability The dataset analysed during the current study is available from the corresponding author upon reasonable request.

Code availability Not applicable.

Author contributions All authors read and approved the final version of this manuscript.

Open Access This article is licensed under a Creative Commons Attribution-NonCommercial 4.0 International License, which permits any non-commercial use, sharing, adaptation, distribution and reproduction in any medium or format, as long as you give appropriate credit to the original author(s) and the source, provide a link to the Creative Commons licence, and indicate if changes were made. The images or other third party material in this article are included in the article's Creative Commons licence, unless indicated otherwise in a credit line to the material. If material is not included in the article's Creative Commons licence and your intended use is not permitted by statutory regulation or exceeds the permitted use, you will need to obtain permission directly from the copyright holder. To view a copy of this licence, visit http://creativecommons.org/licenses/by-nc/4.0/.

\section{References}

1. Seyedian SS, Nokhostin F, Malamir MD. A review of the diagnosis, prevention, and treatment methods of inflammatory bowel disease. J Med Life. 2019;12(2):113-22.

2. Huttenhower C, Kostic AD, Xavier RJ. Inflammatory bowel disease as a model for translating the microbiome. Immunity. 2014;40(6):843-54.

3. Fakhoury M, Negrulj R, Mooranian A, Al-Salami H. Inflammatory bowel disease: clinical aspects and treatments. J Inflamm Res. 2014;7:113-20.

4. A Guideline on Summary of Product Characteristics (SmPC). Directorate-General. European Commission Enterprise and Industry; 2009. p. 29.

5. de Jong ME, Smits LJT, van Ruijven B, den Broeder N, Russel M, Romkens TEH, et al. Increased discontinuation rates of anti-TNF therapy in elderly inflammatory bowel disease patients. J Crohns Colitis. 2020;14(7):888-95.

6. Netherlands Pharmacovigilance Centre Lareb. About Lareb. https ://www.lareb.nl/en/pages/about-lareb. Accessed 2 Jul 2020.

7. (ICH) Medical Dictionary for Regulatory Activities. https://www. meddra.org/. Accessed 14 Apr 2020.

8. CBG Geneesmiddeleninformatiebank College ter Beoordeling van Geneesmiddelen. https://www.geneesmiddeleninformatiebank.nl/ nl/. Accessed 14 Mar 2020

9. AbbVie. Summary of product characteristics, Humira. Wiesbaden: AbbVie Biotechnology GmbH; 2008.

10. Mylan. Summary of product characteristics, azathioprine mylan $25 \mathrm{mg} / 50 \mathrm{mg}$. Bunschoten: Mylan B.V; 2019.

11. Janssen-Cilag. Summary of product characteristics, Remicade. Beerse: Janssen Biologics B.V; 2009.

12. Aspen. Summary of product characteristics, puri-nethol $50 \mathrm{mg}$ tablet. Dublin: Aspen Pharma Trading Limited; 2020.

13. Shire. Summary of product characteristics, mezavant $1200 \mathrm{mg}$. Dublin: Shire Pharmaceuticals Ireland Limited; 2020.

14. Aurobindo. Summary of product characteristics, mesalazine aurobindo $250 \mathrm{mg}$, zetpillen. Baarn: Aurobindo Pharma B.V; 2019.

15. Accord. Summary of product characteristics, methotrexate accord 20 mg, tablets. Haarlem: Accord Healthcare B.V; 2020.
16. Teva. Summary of product characteristics, thiosix $10-20 \mathrm{mg}$. Haarlem: Teva Nederland BV; 2019.

17. BasicPharma. Summary of Product Characteristics, Methotrexate Basic Pharma $5 \mathrm{mg}=0,133 \mathrm{ml}$. Geleen: Basic Pharma Manufacturing B.V; 2020.

18. Takeda. Summary of Product Characteristics, Entyvio $300 \mathrm{mg}$ poeder voor concentraat voor oplossing voor infusie. Vallensbaek Strand: Takeda Pharma A/S; 2018.

19. Janssen-Cilag. Summary of product characteristics, imponi 45 $\mathrm{mg} / 0.45 \mathrm{ml}$ oplossing voor injectie in een voorgevulde pen. Leiden: Janssen Biologicals B.V; 2009.

20. Janssen-Cilag. Summary of product characteristics, STELARA $130 \mathrm{mg}$ concentraat voor oplossing voor infusie. Leiden: Janssen Biologics B.V; 2009.

21. Biemans VBC, Savelkoul E, Gabriels RY, Simsek M, Dijkstra G, Pierik MJ, de Boer NKHL, Hoentjen F, et al. A comparative analysis of tioguanine versus low-dose thiopurines combined with allopurinol in inflammatory bowel disease patients. Aliment Pharmacol Ther. 2020;51(11):1076-86.

22. Coenen MJ, de Jong DJ, van Marrewijk CJ, Derijks LJ, Vermeulen SH, Wong DR, et al. Identification of patients with variants in TPMT and dose reduction reduces hematologic events during thiopurine treatment of inflammatory bowel disease. Gastroenterology. 2015;149(4):907-17.e7.

23. Macaluso FS, Renna S, Maida M, Dimarco M, Sapienza C, Affronti M, et al. Tolerability profile of thiopurines in inflammatory bowel disease: a prospective experience. Scand J Gastroenterol. 2017;52(9):981-7.

24. Calafat M, Mañosa M, Cañete F, Ricart E, Iglesias E, Calvo M, ENEIDA registry of GETECCU, et al. Increased risk of thiopurine-related adverse events in elderly patients with IBD. Aliment Pharmacol Ther. 2019;50(7):780-8.

25. Hindorf U, Johansson M, Eriksson A, Kvifors E, Almer SH. Mercaptopurine treatment should be considered in azathioprine intolerant patients with inflammatory bowel disease. Aliment Pharmacol Ther. 2009;29(6):654-61.

26. de Boer NKH, Peyrin-Biroulet L, Jharap B, Sanderson JD, Meijer $\mathrm{B}$, Atreya I, et al. Thiopurines in inflammatory bowel disease: new findings and perspectives. J Crohns Colitis. 2018;12(5):610-20.

27. Simsek M, Deben DS, Horjus CS, Benard MV, Lissenberg-Witte BI, Buiter HJC, et al. Sustained effectiveness, safety and therapeutic drug monitoring of tioguanine in a cohort of 274 IBD patients intolerant for conventional therapies. Aliment Pharmacol Ther. 2019;50(1):54-65.

28. Hindorf U, Lindqvist M, Peterson C, Soderkvist P, Strom M, Hjortswang $\mathrm{H}$, et al. Pharmacogenetics during standardised initiation of thiopurine treatment in inflammatory bowel disease. Gut. 2006;55(10):1423-31.

29. Hindorf U, Lindqvist M, Hildebrand H, Fagerberg U, Almer S. Adverse events leading to modification of therapy in a large cohort of patients with inflammatory bowel disease. Aliment Pharmacol Ther. 2006;24(2):331-42.

30. Qiu Y, Mao R, Zhang SH, Li MY, Guo J, Chen BL, et al. Safety profile of thiopurines in crohn disease: analysis of 893 patientyears follow-up in a Southern China Cohort. Medicine (Baltimore). 2015;94(41):e1513.

31. Rubin DT. Recent research on joint pain and arthritis in patients with inflammatory bowel disease. Gastroenterol Hepatol (N Y). 2017;13(11):688-90.

32. Hazell L, Shakir SA. Under-reporting of adverse drug reactions: a systematic review. Drug Saf. 2006;29(5):385-96. 